DOI: $10.4274 /$ jarem.galenos.2021.85057

J Acad Res Med 2021;11(1):97-101

\title{
Targeted Follow-up of Incidental Lung Nodules: Will the New Nodules in Unscanned Regions Be Missed?
}

\author{
(1) Terman Gümüş1, (1) Gökhan Uçar2 \\ ${ }^{1}$ Koç University Faculty of Medicine, Department of Radiology, İstanbul, Turkey \\ ${ }^{2}$ St. Walburga Hospital, Radprax MVZ, Südwestfalen, Germany
}

Cite this article as: Gümüş T, Uçar G. Targeted Follow-up of Incidental Lung Nodules: Will the New Nodules in Unscanned Regions Be Missed?. J Acad Res Med 2021;11(1):97-101

\begin{abstract}
Objective: Targeted computed tomography (TCT) scans a limited part or those parts of the lung with nodules that require follow-up. In order to apply targeted tomography safely, it is important to know the frequency of newly appearing nodules in unscanned regions of lung. We aimed to evaluate the frequency and importance of new pulmonary nodules that appear in patients followed-up for nodules according to Fleischner society guidelines.

Methods: A total of 117 patients (women: 54; men: 63; mean age: 55 \pm 14 years; range: 30-88 years) who were followed-up for 265 lung nodules were included in this study. The inclusion criteria was presence of at least 1 nodule that was followed-up for at least 6 months with CT. Patients with calcified nodules or known malignancy during initial CT were excluded.

Results: The median follow-up time was 22 months (range: 6-80 months) and the median number of follow-up CT scans was 2 (range: 1-5). New nodules appeared only in 6\% (7/117) of the patients, of which 5 had nodules that disappeared or decreased in size during follow-up.

Conclusion: For patients who were followed-up for pulmonary nodules, the frequency of new nodule formation was low. This should encourage the use of TCT for nodule follow-up. With an appropriate TCT follow-up schedule, patients will receive reasonably low radiation levels without affecting their management.
\end{abstract}

Keywords: Lung nodules follow-up, chest CT, targeted computed tomography, low-dose CT

\section{INTRODUCTION}

Lung nodules are commonly detected on chest computed tomography (CT) scans. Although most of these nodules are benign, there is currently no safe method to predict the malignant potential of the nodules unless they are followed-up (1). Many patients with solid lung nodules require follow-up with CT for 2 years $(2,3)$. Subsolid lung nodules may even require follow-up for as long as 3-5 years (4). After initial CT, depending on the risk factors of the patient, as well as the size and configuration of the nodules, patients may require 2-6 control CT examinations (2).
Repeated CT examinations increase the radiation exposure of patients. Particularly, for younger individuals, the potential harms of radiation exposure via repeated diagnostic imaging may exceed its benefits. A number of studies have attempted to lower CT radiation doses (5-7). Targeted CT (TCT) scans a limited part or those parts of the lung with nodules that require follow-up. In addition to low-dose CT techniques, TCT for lung nodules may reduce the dose delivered to a patient by as much as $80 \%$ (8).

However, before TCT for lung nodule follow-up can gain wide acceptance, the potential of new nodule formation in unscanned lung regions must be defined. Thus, this study aimed to evaluate

ORCID IDs of the authors: T.G. 0000-0001-5882-3083; G.U. 0000-0002-2503-5061.

Corresponding Author/Sorumlu Yazar: Terman Gümüş, E-mail: termang@gmail.com
Received Date/Geliş Tarihi: 14.02.2021 Accepted Date/Kabul Tarihi: 20.03.2021

(C) Copyright 2021 by University of Health Sciences Turkey, Gaziosmanpaşa Training and Research Hospital. Available on-line at www.jarem.org 
the frequency and importance of new pulmonary nodules in patients followed-up for incidental lung nodules.

\section{METHODS}

\section{Patient Selection and Imaging}

The study protocol was reviewed and approved by the Koç University Ethics Committee (approval number: 2014.073.IRB2.019, approval date: 29.04.2014), which waived the requirement of informed consent. All lung CT medical reports in our radiology database between January 2008 and December 2010 were searched retrospectively.

We included a total of 117 patients who had at least 1 nodule and control CT imaging (no sooner than 6 months). Patients with calcified nodules or a known malignancy during initial CT were excluded. Of the 117 patients, 62 had a smoking history of over 20 pack/year, 41 had a smoking history between 10 and 19 pack/year, and 12 had a smoking history between 4 and 9 pack/year.

For all patients, CT was performed using $2 \times 64$ Somatom Definition and 2×128 Somatom Definition Flash Multidetector CT (Siemens Healthcare, Erlangen, Germany). The entire chest starting from the lung apices down to posterior costophrenic sulci was scanned with $0.625 \mathrm{~mm}$ collimation, 80-120 kVp and 40-120 mAs. Images were reconstructed with $1.5 \mathrm{~mm}$ section thickness.

All nodules identified from previous reports were re-evaluated using a picture archiving and communication system (Centricity PACS, General Electric Medical Systems, Milwaukee, WI) by two experienced radiologist who have over 10 years of experience in interpreting CT scans of the chest. All discrepancies were resolved by consensus. The number and mean dimensions of the nodules were determined. Follow-up duration was determined according to Fleischner society guidelines (2). Location of the nodules was defined on the basis of pulmonary lobes. The margins of the nodules were classified as well- or ill-defined with a smooth or spiculated border. The nodules were also classified as solid or subsolid. New nodules were recorded and evaluated on the basis of size, location, margin characteristics and density. Changes in new nodules were evaluated when available. In addition, all patients mediastinum and bones were also evaluated for potential pathologies.

\section{Statistical Analysis}

Statistical analysis was performed using the Statistical Package for the Social Sciences, Version 22 (SPSS Inc., Chicago, Illinois, USA). Results were expressed as medians (ranges).

\section{RESULTS}

We included a total of 117 patients (women: 54; men: 63; mean age: $55 \pm 14$ years; range: $30-88$ years) with 265 nodules on initial CT scans (Table 1). Median follow-up time was 22 months (range: 6-80 months) (Table 2) and median number of follow-up CT scans was 2 (range: 1-5).

Of the 265 nodules, 246 (93\%) were solid, 19 (7\%) were subsolid; $254(96 \%)$ nodules had smooth borders, while 11 (4\%) had irregular

Table 1. Selected baseline characteristics of the study group

Age

30-39

40-49

$50-59$

$60-69$

$70-79$

$\geq 80$

Gender

Female

Male

\section{Table 2. Follow-up time for initial nodules}

Follow-up time

6-12 months

$34(13.3 \%)$

13-18 months

$60(23.5 \%)$

19-24 months

$58(22.6 \%)$

$>24$ months

$104(40.6 \%)$

borders. Of the 117 patients, 57 (49\%) had a single nodule, 22 (19\%) had 2 nodules, 14 (12\%) had 3 nodules and $24(21 \%)$ had $>3$ nodules.

Regarding the location of the nodules, $46(17.4 \%)$ were in the right upper lobe, $26(10 \%)$ were in the middle lobe, $89(33 \%)$ were in the right lower lobe, 36 (14\%) were in the left upper lobe and $68(26 \%)$ were in the left lower lobe.

Among the 265 nodules, 252 (95\%) were stable, 5 (2\%) increased in size and 8 (3\%) decreased in size on follow-up. Two of the 5 nodules that increased in size were proven malignant by histopathological evaluation and removed by lobectomy. One of them decreased in size on subsequent follow-up and the other two maintained a stable size.

A total of 16 new nodules were detected in 7 (6\%) patients. Subgroup analysis for these new nodules revealed the following: In 3 patients, the nodules disappeared and, in 2 patients, the nodules decreased in size (from $7 \mathrm{~mm}$ to $3 \mathrm{~mm}$ in 1 patient and from $12 \mathrm{~mm}$ to $9 \mathrm{~mm}$ in the other patient). Two patients had been lost to follow-up: One patient had new nodules of $2 \mathrm{~mm}$ and 3 $\mathrm{mm}$ and the other had new nodules of $3 \mathrm{~mm}$ and $5 \mathrm{~mm}$ and could not be followed-up (Figure 1). All patients with new nodules are summarised in Table 3. During follow-up in none of the patients, new lymphadenopathies or bone lesions were detected.

\section{DISCUSSION}

Follow-up of lung nodules and screening for lung cancer are routine procedures in many countries. Although many studies have attempted to reduce patient doses during lung CT, TCT for nodule follow-up has not been extensively discussed. After initial CT for patients with fewer than 3 nodules or nodules that 


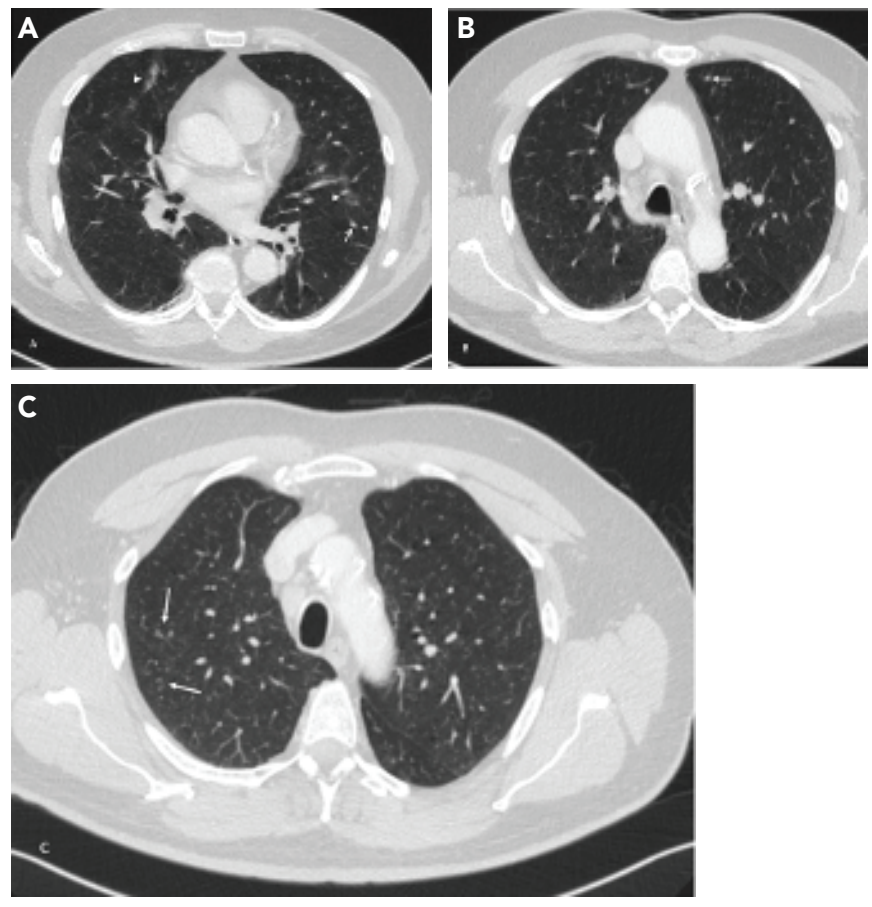

Figure 1. A 55-year-old male patient who has been followed-up for $3 \mathrm{~mm}$ and $5 \mathrm{~mm}$ nodules underwent computed tomography (CT) imaging for $22^{\text {th }}$ month control. Non-contrast CT image shows (A) $5 \mathrm{~mm}$ and (B) $3 \mathrm{~mm}$ new appearing nodules (arrows) in the left upper lobe. Note that there is focal ground glass infiltration (arrowhead) in both upper lobes. (C) Right upper lobe image shows centrilobular nodules, which were absent in previous CT. Unless we lost contact with patient, these secondary findings may be suggestive of an infectious origin of these two nodules are in the same lobe, TCT may be the most effective method for reducing the radiation dose delivered to a patient.

In this study, we determined the frequency of new nodules and their outcomes, which may be a major drawback for using TCT. Assuming that TCT was used for our study group instead of conventional whole lung scanning, only 7 of the 117 patients were found to have new nodules. Five patients' nodules disappeared or decreased in size during follow-up. Only 2 (1.7\%) of the 117 patients would have nodules that may have been missed with TCT. These nodules were smaller than $5 \mathrm{~mm}$ and had smooth contours. None of these nodules were proven malignant.

Our median follow-up period was nearly 2 years, which should have been sufficient to detect new nodule potential in a population that was being followed-up for incidental lung nodules. This showed that at least $97.3 \%$ patients who were followed-up for incidental lung nodules had unnecessary whole lung CT scanning. We lost contact with 2 patients who had new nodules. For patients who had fewer than 4 nodules, follow-up CT within a year using TCT appears feasible. However, this technique still requires validation prospectively in larger groups.

These findings are important because patients may be followedup with significantly reduced radiation exposure. TCT will also save the scanner tube life, which will reduce the expenses of clinics. In addition, using TCT would shorten interpretation and report times due to the decreased anatomical coverage.

Follow-up with TCT may be impractical for multiple nodules; nevertheless, it may be practical to use TCT for less than four nodules. Among our patients, $79.5 \%$ had fewer than 4 nodules and $48.7 \%$ had only 1 nodule. These findings suggest that a large

\section{Table 3. Characteristics of new nodules}

\begin{tabular}{|c|c|c|c|c|c|c|c|c|}
\hline Patients & Nodule & Gender & Age & Location & Size $(\mathrm{mm})$ & Density & Margin & Follow-up \\
\hline Patient 1 & Nodule 2 & Female & 75 & left upper lobe & 2 & solid & smooth & na \\
\hline Patient 3 & Nodule 1 & \multirow{2}{*}{ Male } & \multirow{2}{*}{55} & left upper lobe & 5 & solid & smooth & na \\
\hline Patient 3 & Nodule 2 & & & left upper lobe & 3 & solid & smooth & na \\
\hline Patient 4 & Nodule 2 & \multirow{4}{*}{ Female } & \multirow{4}{*}{51} & left upper lobe & 6 & subsolid & smooth & disappeared \\
\hline Patient 4 & Nodule 3 & & & left upper lobe & 3 & solid & smooth & disappeared \\
\hline Patient 4 & Nodule 4 & & & left upper lobe & 3 & solid & smooth & disappeared \\
\hline Patient 4 & Nodule 5 & & & right upper lob & 4 & subsolid & smooth & disappeared \\
\hline Patient 5 & Nodule 1 & Female & 61 & right upper lob & 5 & solid & irregular & disappeared \\
\hline Patient 6 & Nodule 1 & Male & 35 & left upper lobe & 12 & solid & smooth & decreased in size \\
\hline Patient 7 & Nodule 1 & Female & 71 & right middle lobe & 7 & solid & irregular & decreased in size \\
\hline
\end{tabular}


number of patients who are followed-up for incidental nodules will experience the benefits of TCT.

There have been discussions regarding the lowering of the age for lung nodule screening. Screening younger age groups may increase the chance of early lung cancer detection, which should contribute to improvement in survival rates $(9,10)$. The major drawback of lowering the population age for lung screening is the possible harmful effects of ionising radiation, since younger people are more sensitive to the effects of ionising radiation (11). When TCT is used for follow-up, high-risk populations may be screened at a younger age. After an initial low dose of whole lung CT scan, patients with fewer than 4 nodules may be followed-up using TCT at very low doses. Further studies must be conducted to determine the age groups and risk factors for screening people who are younger than 55 years. Using TCT for nodule follow-up may make earlier screening safe and applicable.

In this study, all the 16 new nodules detected were sub-centimetric, except one. Most of these nodules probably had an infectious origin and had decreased in size or disappeared on follow-up. One patient showed 8 new nodules on her second follow-up CT; however, 6 months later during her next control CT, all those nodules had disappeared. One patient had a 5-mm nodule that disappeared within 15 months (Figure 2) and another patient had a 2-mm nodule that disappeared within 12 months. One patient was being followed-up for left upper lobe nodules that were most likely of granulomatous origin and a new 12-mm nodule was found in the same lobe. This new nodule decreased to $10 \mathrm{~mm}$ within 20 months, which suggests that it is from a benign origin (Figure 3). One patient was being followed-up for 6 nodules (size range: 4-6 $\mathrm{mm}$ ) and a new $7 \mathrm{~mm}$ nodule was found, which decreased to 5 $\mathrm{mm}$ within 9 months (Figure 4).

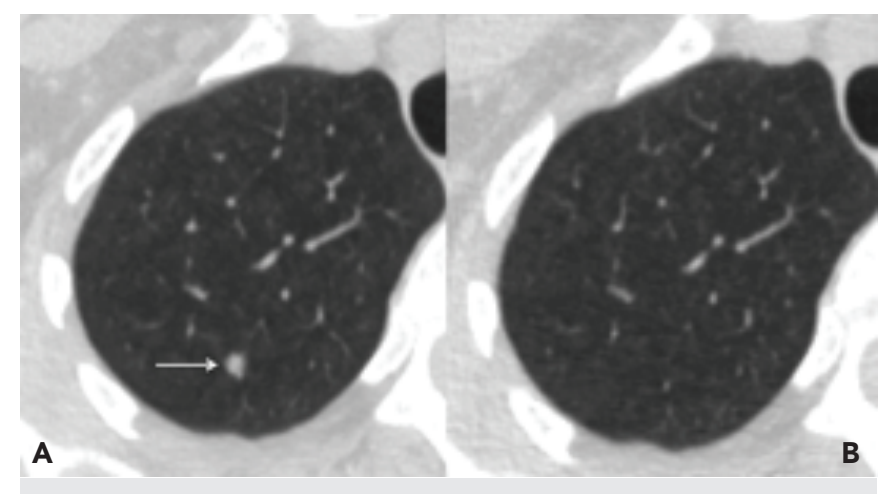

Figure 2. A 61-year-old female who has been followed-up for a 6 $\mathrm{mm}$ nodule in the left lower lobe underwent non-contrast control computed tomography imaging, (A) which showed a $5 \mathrm{~mm}$ new nodule (arrow) in the right upper lobe. (B) 15 months later, this 5 $\mathrm{mm}$ nodule disappeared, thus revealing its benign nature

\section{Study Limitations}

The limitations of our study included the retrospective nature of the study. Also, we could not follow-up 2 patients with new

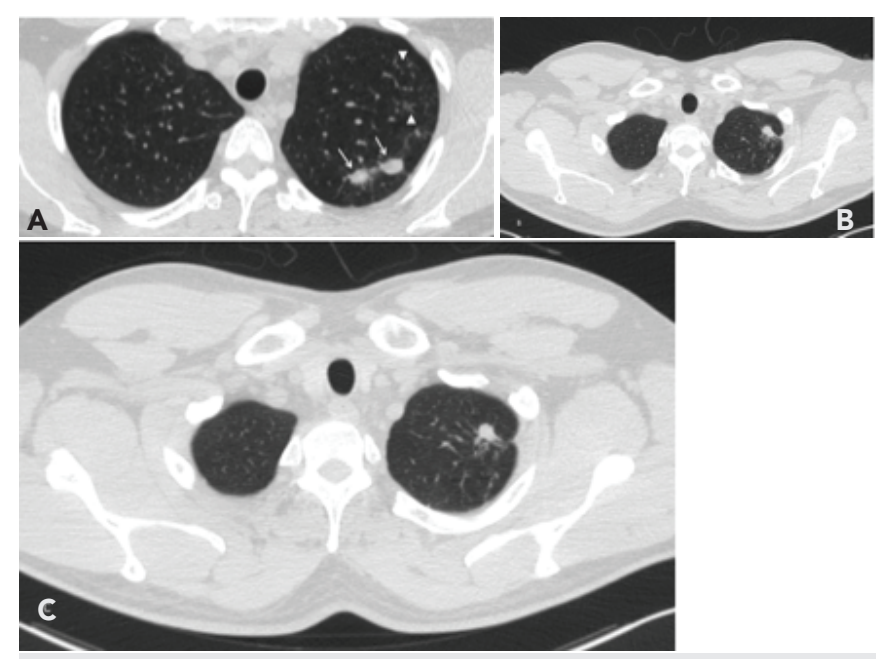

Figure 3. A 35-year-old male. (A) Non-contrast computed tomography (CT) image shows two nodules (arrows) that were decided to be followed-up. Anteriorly centrilobular nodules (arrowhead) may suggest an active infectious process. (B) $6^{\text {th }}$ month control CT of left upper lobe shows new nodule in the area where centrilobular nodules were previously seen. (C) Follow-up $\mathrm{CT}$ after 20 months shows a decrease in size of the nodule

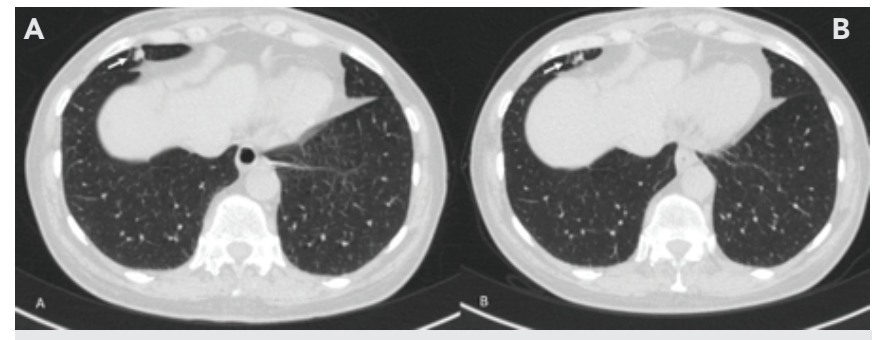

Figure 4. A 71-year-old female who has been followed-up for subcentimetric six nodules underwent non-contrast control computed tomography imaging and (A) showed new $7 \mathrm{~mm}$ nodule (arrow) in middle lobe. (B) 9 months later, the nodule decreased in size

nodules because we lost contact with them. In our study group, with 256 nodules, the malignancy rate was low (0.78\%). Most of the patients who have nodules with malign CT features undergo biopsy or surgery after initial CT instead to be followed-up. This could explain the low malignancy rate seen in our cohort.

In this study, we did not define whether TCT was practical for covering the targeted nodule or nodules. One technique proposed for TCT for nodule follow-up claimed that all nodules during follow-up were scanned in their entirety (8). In addition, we could not compare the radiation doses between TCT and conventional whole lung CT. Our primary aim was to provide information regarding new nodule frequency in a population that was followed-up for lung nodules. The low frequency of new nodule formation should instigate the need for further studies.

Determination of the application method and patient selection criteria for TCT were beyond the scope of this study. To resolve these issues, prospective studies will be required for TCT for lung nodule follow-up. 


\section{CONCLUSION}

In patients who were followed-up for incidental nodules, the frequency of new nodule formation seems to be low. This should encourage the use of TCT for nodule follow-up. With an appropriate TCT follow-up schedule, patients will administered reasonably low radiation levels without affecting their management.

Ethics Committee Approval: The study protocol was reviewed and approved by the Koç University Ethics Committee (approval number: 2014.073.IRB2.019, approval date: 29.04.2014).

Informed Consent: Retrospective study.

Peer-review: Externally peer-reviewed.

Author Contributions: Concept - T.G., G.U.; Design - T.G.; Data Collection and/or Processing - T.G., G.U.; Analysis and/or Interpretation - T.G., G.U.; Literature Search - G.U.; Writing - T.G.

Conflict of Interest: The authors have no conflict of interest to declare.

Financial Disclosure: The authors declared that this study has received no financial support.

\section{REFERENCES}

1. Swensen SJ. CT screening for lung cancer. AJR Am J Roentgenol 2002; 179: 833-6.

2. MacMahon H, Naidich DP, Goo JM, Lee KS, Leung AN, Mayo JR, et al. Guidelines for management of incidental pulmonary nodules detected on CT images: From the Fleischner Society 2017. Radiology 2017; 284: 228-43.

3. Slattery MM, Foley C, Kenny D, Costello RW, Logan PM, Lee MJ. Longterm follow-up of non-calcified pulmonary nodules $(<10 \mathrm{~mm})$ identified during low-dose CT screening for lung cancer. Eur Radiol 2012; 22: 19238.

4. Godoy MC, Naidich DP. Overview and strategic management of subsolid pulmonary nodules. J Thorac Imaging 2012; 27: 240-8.

5. Rampinelli C, Origgi $D$, Bellomi M. Low-dose $C T$ : technique, reading methods and image interpretation. Cancer Imaging 2013; 12: 548-56.

6. Marshall HM, Bowman RV, Yang IA, Fong KM, Berg CD. Screening for lung cancer with low-dose computed tomography: a review of current status. J Thorac Dis 2013; 5(Suppl 5): S524-39. doi: 10.3978/j.issn.20721439.2013.09.06.

7. Yanagawa M, Gyobu $T$, Leung AN, Kawai M, Kawata $Y$, Sumikawa $H$, et al. Ultra-low-dose CT of the lung: effect of iterative reconstruction techniques on image quality. Acad Radiol 2014; 21: 695-703.

8. Pearson G, Rozenshtein A, Toy D. (2012, November 25-30). Targeted Follow-up of incidental lung nodules: a practical technique for narrowing craniocaudal coverage resulting in $80 \%$ radiation dose reduction [Paper presentation]. Annual Meeting of the Radiological Society of North America; Chicago IL, USA. Available from: https://archive.rsna. org/2012/12031091.html.

9. Henschke $\mathrm{Cl}$, Yankelevitz DF, Libby DM, Pasmantier MW, Smith JP, Miettinen OS. Survival of patients with stage I lung cancer detected on CT screening. N Engl J Med 2006; 355: 1763-71.

10. Wood DE, Eapen GA, Ettinger DS, Hou L, Jackman D, Kazerooni E, et al. Lung cancer screening. J Natl Compr Canc Netw 2012; 10: 240-65.

11. Shuryak I, Sachs RK, Brenner DJ. Cancer risks after radiation exposure in middle age. J Natl Cancer Inst 2010; 102: 1628-36. 